\title{
Radar absorption, basal reflection, thickness and polarization measurements from the Ross Ice Shelf, Antarctica
}

\author{
Jordan C. HANSON, ${ }^{1,2}$ Steven W. BARWICK, ${ }^{1}$ Eric C. BERG, ${ }^{1}$ Dave Z. BESSON, ${ }^{2,3}$ \\ Thorin J. DUFFIN, ${ }^{1}$ Spencer R. KLEIN,${ }^{4}$ Stuart A. KLEINFELDER, ${ }^{5}$ Corey REED, ${ }^{1}$ \\ Mahshid ROUMI, ${ }^{1}$ Thorsten STEZELBERGER, ${ }^{4}$ Joulien TATAR, ${ }^{1}$ James A. WALKER, ${ }^{1}$ \\ Liang $\mathrm{ZOU}^{1}$
}

\author{
${ }^{1}$ Department of Physics and Astronomy, University of California, Irvine, Irvine, CA, USA \\ ${ }^{2}$ Department of Physics and Astronomy, University of Kansas, Lawrence, KS, USA \\ ${ }^{3}$ Moscow Physics and Engineering Institute, Moscow, Russia \\ ${ }^{4}$ Lawrence Berkeley National Laboratory, Berkeley, CA, USA \\ ${ }^{5}$ Department of Electrical Engineering and Computer Science, University of California, Irvine, CA, USA \\ Correspondence: Jordan C. Hanson <918particle@gmail.com>
}

\begin{abstract}
Radio-glaciological parameters from the Moore's Bay region of the Ross Ice Shelf, Antarctica, have been measured. The thickness of the ice shelf in Moore's Bay was measured from reflection times of radio-frequency pulses propagating vertically through the shelf and reflecting from the ocean, and is found to be $576 \pm 8 \mathrm{~m}$. Introducing a baseline of $543 \pm 7 \mathrm{~m}$ between radio transmitter and receiver allowed the computation of the basal reflection coefficient, $R$, separately from englacial loss. The depth-averaged attenuation length of the ice column, $\langle L\rangle$ is shown to depend linearly on frequency. The best fit $\left(95 \%\right.$ confidence level) is $\langle L(\nu)\rangle=(460 \pm 20)-(180 \pm 40) \nu \mathrm{m}\left(20 \mathrm{~dB} \mathrm{~km}^{-1}\right)$, for the frequencies $\nu=[0.100-0.850] \mathrm{GHz}$, assuming no reflection loss. The mean electric-field reflection coefficient is $\sqrt{R}=0.82 \pm 0.07(1.7 \mathrm{~dB}$ reflection loss) across [0.100-0.850] GHz, and is used to correct the attenuation length. Finally, the reflected power rotated into the orthogonal antenna polarization is $<5 \%$ below $0.400 \mathrm{GHz}$, compatible with air propagation. The results imply that Moore's Bay serves as an appropriate medium for the ARIANNA high-energy neutrino detector.
\end{abstract}

KEYWORDS: ice/ocean interactions

\section{INTRODUCTION}

The vast Antarctic ice sheet has become important to highenergy neutrino physics in recent years (Barwick, 2007; ANITA Collaboration, 2010; Klein, 2012; Kravchenko and others, 2012; IceCube Collaboration, 2013), motivated by the convenient properties of glacial ice, including optical and radio-frequency (RF) dielectric properties. High-energy cascades induced by neutrinos emit Cherenkov photons; photons with $350-500 \mathrm{~nm}$ wavelengths can propagate 10 $100 \mathrm{~m}$ in Antarctic ice before being detected by photomultiplier tubes (AMANDA Collaboration, 2006). Similarly, at energies $0.1 \mathrm{EeV}$, neutrinos begin to produce measurable Askaryan pulses (Askaryan, 1962), a form of coherent Cherenkov radiation in the RF regime. Moore's Bay, part of the Ross Ice Shelf, presents an attractive target volume for studying these particle interactions, because the radiation experiences minimal attenuation in the cold ice, and can be reflected back towards the surface by the ocean. Knowledge of the dielectric properties of the ice shelf is required to build such an experiment.

By transmitting a $\sim 1 \mathrm{~ns}$ pulse through a transmitting antenna downwards through the ice shelf, and recording the reflections from the oceanic interface, bulk ice attenuation and the reflection coefficient of the interface can be inferred. Using reflected radio pulses to study ice sheets and shelves is known as radio-echo sounding, and has been used to study glaciers and ice shelves in various locations on the Ross Ice Shelf (RIS) and the high plateau (Neal, 1979, 1982;
Besson and others, 2008; Allison and others, 2012; Fretwell and others, 2013).

Basal reflection in Moore's Bay has been studied previously. Neal $(1979,1982)$ reported on the RIS, using a $60 \mathrm{~ns}$ wide, $60 \mathrm{MHz}$ pulse, recording the returned power vs location. Flights $1 \mathrm{~km}$ above the RIS were performed, including several points over Moore's Bay. Basal reflection coefficients were derived in $10 \mathrm{~dB}$ increments, assuming no losses from dust or other impurities, for contours across the shelf. Moore's Bay produces reflection coefficients near the Fresnel limit $(-0.82 \mathrm{~dB}$, or $\sim 0.91$ for the electric field), and two explanations were offered. First, Moore's Bay is far from brine percolation zones that are traced from the grounding line to the shelf front, which are correlated with ice velocity. Second, the melt rate near the grounding line for basal ice prevents the formation of an abrupt basal layer of saline ice, and instead replaces glacial ice with saline ice over time. The freeze-on of saline ice at the shelf bottom does occur; however, these regions are far from the location of the Antarctic Ross Ice-Shelf ANtenna Neutrino Array (ARIANNA), and the average accumulation rate of bottom saline ice is only $0.3 \pm 0.1 \mathrm{~m} \mathrm{a}^{-1}$ in the east RIS (Rignot and others, 2013).

Neal (1982) showed that two parameters besides peak power can be extracted from the data. First, the width of the peak power distribution for a specific location pertains to vertical roughness at the oceanic interface. Second, the spatial correlation of power measurements reveals horizontal correlation lengths for roughness. These measurements must 
be compared to theoretical distributions of the same parameters, from the theory of rough-surface scattering (Dookayka, 2011). The most general statistical surface, with the fewest parameters, was chosen: a Gaussian surface roughness described by normal fluctuations about a mean depth, and a specified horizontal correlation length. Neal reports vertical root-mean-square $(\mathrm{rms})$ of $3 \mathrm{~cm}$ at the ocean/ ice boundary, spread over correlation lengths of $27.5 \mathrm{~m}$, $30 \mathrm{~km}$ east of Ross Island. The results make no use of absolute power measurements, and thus are independent of assumptions of RF absorption.

To measure the reflection coefficient separately from englacial loss, the transmitting and receiving antennas may be separated by a baseline comparable to the shelf depth. The signal path is longer with the baseline, providing different absorption but the same reflection loss (Hanson, 2011 , 2013). By comparing total loss in the set-up with and without a baseline, absorption and reflection loss can be measured separately. A map of the site studied in this work is shown in Figure 1.

\section{RF attenuation length}

The amplitude of an electric field decreases by 1/e after propagating one attenuation length. For an electromagnetic plane wave travelling through a dielectric medium with a complex index of refraction $n=n^{\prime}-i n^{\prime \prime}$, the electric field is

$$
\mathbf{E}=\mathbf{E}_{0} \exp (-i(n k x-\omega t))=\mathbf{E}_{0}^{\prime} \exp \left\{-n^{\prime \prime} k x\right\}
$$

The electric field attenuation length is then $\left(n^{\prime \prime} k\right)^{-1}=L(\omega$ is the angular frequency). When measured over a volume of material with varying dielectric absorption, the attenuation length is averaged over the effect of depth on the dielectric constant $\epsilon=\epsilon^{\prime}-i \epsilon^{\prime \prime}$, and in turn the loss tangent, $\tan \delta=$ $\epsilon^{\prime \prime} / \epsilon^{\prime}$. If $\tan \delta \ll 1$, it can be shown that

$$
\begin{aligned}
\langle L\rangle^{-1} & =(\pi \nu / c) \sqrt{\epsilon^{\prime}} \tan \delta\left(\mathrm{m}^{-1}\right) \\
N_{L}\left(\mathrm{~dB} \mathrm{~km}{ }^{-1}\right) & =8686.0\langle L[m]\rangle^{-1}
\end{aligned}
$$

Equation (3) is the conversion from attenuation length to absorption loss, $N_{L}$. The Debye model shows that $\nu \tan \delta$ is approximately constant, provided the frequency is far from any molecular resonances (this is true for $0.1-1 \mathrm{GHz}$ ). Additionally, $\epsilon^{\prime}$ (in ice) is constant for the bandwidth 0.1$2 \mathrm{GHz}$. Thus, frequency dependence in $\langle L\rangle$ is attributed to other effects, such as acids and sea-salt impurities (Bogorodsky and others, 1985; Matsuoka and others, 2012).

\section{Reflection coefficient}

Under the Debye model, with a single relaxation time, the ice conductivity is $\sigma=2 \pi \epsilon_{0} \epsilon^{\prime} \nu \tan \delta \approx 10 \mu \mathrm{S} \mathrm{m}^{-1}$ at $100 \mathrm{MHz}$ (Dowdeswell and Evans, 2004). By comparison, sea water has a conductivity of a few $\mathrm{Sm}^{-1}$, with a skin depth of $30 \mathrm{~mm}$, at $60 \mathrm{MHz}$ (Dowdeswell and Evans, 2004; Somaraju and others, 2006). The reflection coefficient for the electric fields $(\sqrt{R}$, where $R$ refers to power) is given by $\left(n_{1}-n_{2}\right) /\left(n_{1}+n_{2}\right)$, given the complex $n_{1}=\sqrt{\epsilon_{1}}$ and $n_{2}=\sqrt{\epsilon_{2}}$ for the dielectric and conductive media, respectively.

$$
\begin{aligned}
& \lim _{\tan \delta_{2} \gg 1, \tan \delta_{1} \rightarrow 0}|\sqrt{R}|=\frac{1-n_{2} / n_{1}}{1+n_{2} / n_{1}} \\
&=\frac{1-\sqrt{\alpha} \mathrm{e}^{-i \delta_{2} / 2}}{1+\sqrt{\alpha} \mathrm{e}^{-i \delta_{2} / 2}}=\left(\frac{1+\alpha-\sqrt{2 \alpha}}{1+\alpha+\sqrt{2 \alpha}}\right)^{1 / 2}
\end{aligned}
$$

Equation (4) demonstrates that $|\sqrt{R}| \rightarrow 1$, where $n_{1}$ refers to

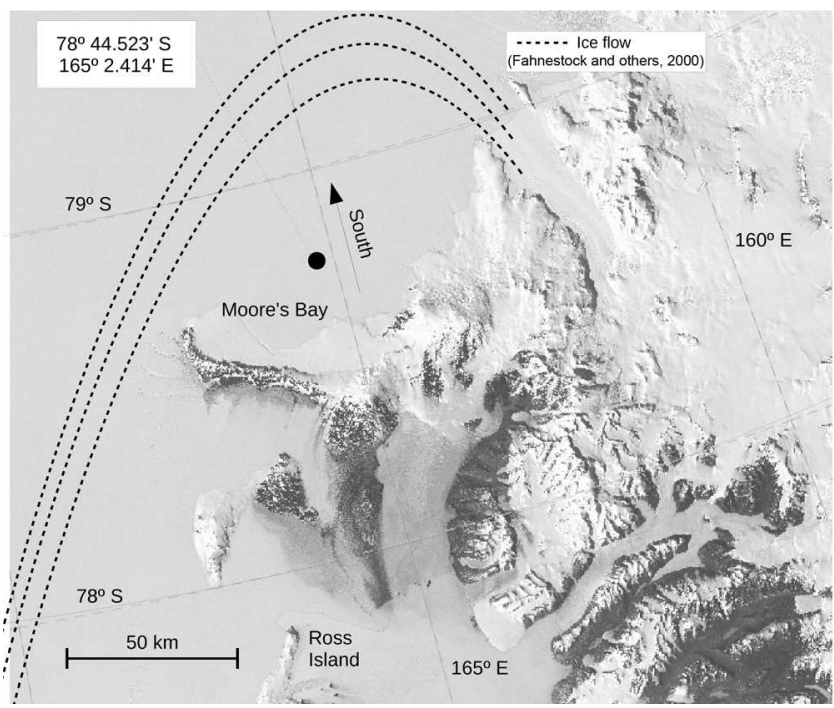

Fig. 1. The site studied in this work is marked with the black circle. Moore's Bay is the area south of Ross Island, enclosed by Minna Bluff. The satellite data alim ade available by the US Geological Survey (USGS, 2012). The main ice flowlines are illustrated with dashed lines. Fahnestock (2000) provides further analysis and discussion.

the ice and $n_{2}$ refers to the ocean, given the limits $\tan \delta_{2} \gg 1$, and $\tan \delta_{1} \approx 0$, and $\alpha=\epsilon_{2}^{\prime \prime} / \epsilon_{1}^{\prime}$. In Eqn (4), the fact that $\delta_{2} \rightarrow \pi / 2$ has been used. Equation (4) is completely general as long as the limit is satisfied. The right-hand side has a global minimum at $\alpha=1$, or $\epsilon_{2}^{\prime \prime}=\epsilon_{1}^{\prime}$, corresponding to a minimum electric field reflection coefficient of $\sqrt{R}_{\text {min }} \approx 0.41$. Realistic values for both ice and sea water indicate $\alpha$ ranges from 20 to 30, depending on the salinity and temperature of the sea water (Dowdeswell and Evans, 2004; Somaraju and others, 2006). Neal (1979) suggested that the reflection coefficient in Moore's Bay is approximately $-0.82 \mathrm{~dB}$, or $\sqrt{R}=0.91$, based on the properties of the sea water beneath the RIS. These upper and lower bounds form an allowed range of $\sqrt{R}=0.41-0.91$.

In addition to vertical radio echoes, measurements were taken with a baseline distance between transmitter and receiver, introducing a new overall path length. In this work, these measurements are named angled bounce studies. For the angled bounce studies reported here, Eqn (5) shows that the reflected power limits to the expression for normal incidence (for s-polarized waves). Also, the initial transmission angle from normal is reduced, because the upper firn layer bends the transmitted pulse downward (to $\theta \approx 30^{\circ}$ ), given the initial antenna orientation of $45^{\circ}$. Ignoring the cosine dependence in Eqn (5) amounts to a $1-5 \%$ correction, depending on $n_{2}$ :

$$
\sqrt{R} \approx \frac{n_{1}\left(1-\theta^{2} / 2\right)-n_{2}\left(1-\frac{1}{2}(\epsilon \theta)^{2}\right)}{n_{1}\left(1-\theta^{2} / 2\right)+n_{2}\left(1-\frac{1}{2}(\epsilon \theta)^{2}\right)} \approx \frac{n_{1}-n_{2}}{n_{1}+n_{2}}
$$

\section{Ice thickness calculation}

The upper $60-70 \mathrm{~m}$ of the ice shelf is firn with density $\approx 0.4 \mathrm{~g} \mathrm{~cm}^{-3}$ near the surface (Gerhardt and others, 2010). This result is in agreement with the value $0.36 \mathrm{~g} \mathrm{~cm}^{-3}$ from Dowdeswell and Evans (2004). Looyenga's equation, $n_{\text {ice }}$ and the firn surface density predict the firn index to be $n_{\text {firn }} \approx 1.3$. This value was confirmed with pulse propagation timing at the surface, over a distance of $543 \pm 7 \mathrm{~m}$ (see 


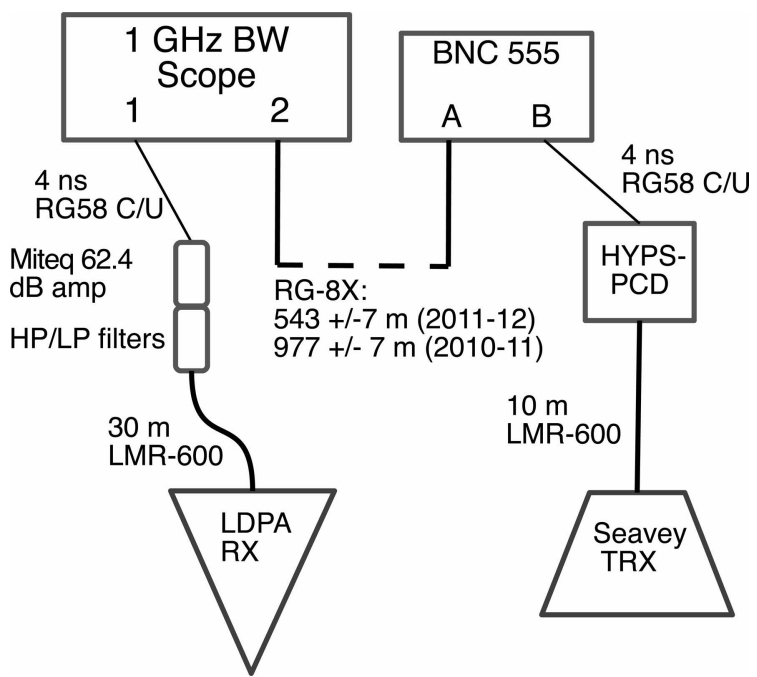

Fig. 2. The general set-up of the radio-sounding experiments. Measured and physical time delays are shown in Table 2.

below for detail). From the pulse arrival time, the implied wave speed indicated an index of $n_{\text {surf }}=1.29 \pm 0.02$ (Hanson, 2013).

The density and thus the index of refraction has an exponential depth dependence, according to the Schytt model:

$$
\begin{aligned}
& n(z)=1.78=n(z \geq 67 m) \\
& n(z)=n_{0}+p \exp (-z / q)(z<67 m)
\end{aligned}
$$

In Eqn (7), $n_{0}=1.86, p=-0.55$ and $q=35.4 \mathrm{~m}$, with the upper layer density $\rho \approx 0.4 \mathrm{~g} \mathrm{~cm}^{-3}$, and $z>0$ for increasing depth. A different model with a constant firn correction (to sounding propagation times) and no exponential density profile yields shelf depths consistent within errors (Gerhardt and others, 2010). Equations (6) and (7) are based on measurements taken at Williams Field near McMurdo station (Schytt, 1958; Barrella and others, 2011). Given the measured physical delay between pulse and reflection, $\Delta t$, the shelf depth can be obtained from integrating over the total path length $d$ (Eqns (8) and (9)). Error propagation yields Eqn (10), where $D_{\mathrm{f}}=67 \pm 10 \mathrm{~m}$ is the firn depth (Dowdeswell

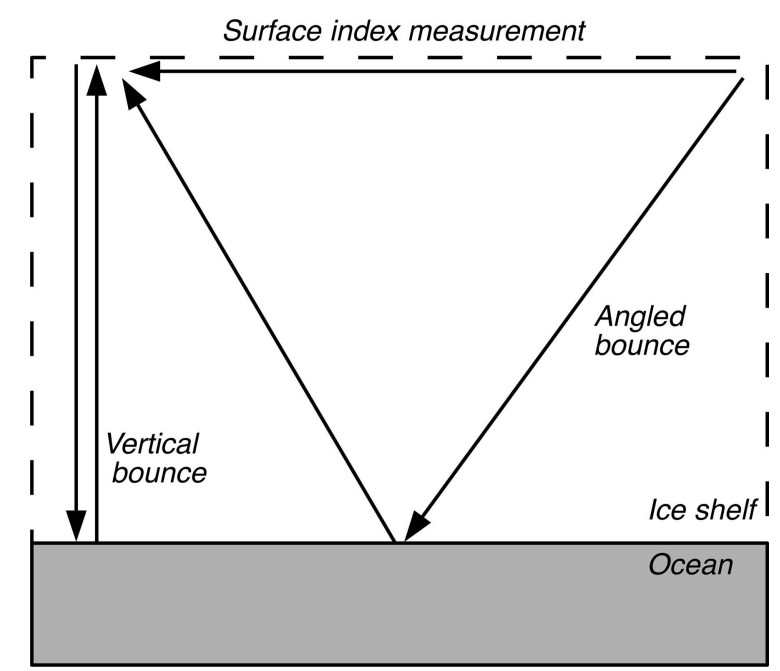

Fig. 3. The vertical and angled bounce tests. The surface propagation set-up was used to derive the surface index of refraction, $n_{\text {surf }}$.
Table 1. The various experimental configurations used, by year, for the data in this work. S stands for Seavey horn, and L stands for LDPA (log-periodic dipole array)

\begin{tabular}{llll}
\hline Year & Vertical/Angled & Ant. (TX/RX) & $G_{1} G_{2}$ \\
\hline 2010 & Vertical & $\mathrm{L} / \mathrm{L}$ & 1.0 \\
2011 & Vertical & $\mathrm{S} / \mathrm{L}$ & 1.0 \\
2011 & Angled & $\mathrm{S} / \mathrm{L}$ & 0.5 \\
2011 & Vertical & $\mathrm{L} / \mathrm{L}$ & 1.0 \\
& & & \\
\hline
\end{tabular}

and Evans, 2004). A density profile for the RIS in figure 2 of the latter reference is consistent with this model.

$$
\begin{gathered}
\frac{c \Delta t}{2}=\int_{0}^{d_{\text {ice }}} n(z) \mathrm{d} z \\
d_{\text {ice }}=\frac{c \Delta t}{2 n}-\frac{D_{\mathrm{f}}\left(n_{0}-n\right)}{n}+\frac{q p}{n}\left(\mathrm{e}^{-D_{\mathrm{f}} / q}-1\right) \\
\sigma_{d, \text { ice }}=\sqrt{\left(\frac{\sigma_{t} C}{2 n}\right)^{2}+\left(\frac{\sigma_{n} c \Delta t}{2 n^{2}}\right)^{2}+k \sigma_{D \mathrm{f}}^{2}} \\
\approx \frac{c}{2 n} \sqrt{\left(\frac{\sigma_{n}}{n}\right)^{2} \Delta t^{2}+\sigma_{t}^{2}}
\end{gathered}
$$

The fractional difference between $n_{0}$ and $n$ is small, and $\exp \left(-D_{\mathrm{f}} / q\right)$ is small, so $k$ turns out to be of order $10^{-2}$. The term in Eqn (10) involving $k$ is a factor of 10 below the others so it may be dropped. For similar reasons, cross-terms involving firn properties and $\sigma_{n}$ have been neglected.

\section{EXPERIMENTAL TECHNIQUE}

The experimental set-up is shown in Figure 2, with additional detail in Table 1. Figure 3 shows the vertical and angled bounces. To create broadband RF pulses, a 1 ns wide, $1-2.5 \mathrm{kV}$ pulse was delivered from the HYPS Pockels Cell Driver (PCD) to a transmitting antenna, and the reflection is received by a second antenna. The PCD and the $1 \mathrm{GHz}$ bandwidth oscilloscope (Tektronix TDS540A in 2010, Agilient HP54832D thereafter) were triggered with a tunable delay generator (Berkeley Nucleonics 555 2-port). From the programmed delay, reflection time and relevant cable delays, the shelf depths were derived for each season. The RG-8X cable between port A of the BNC 555 and the oscilloscope enabled the introduction of a long baseline between the antennas.

Voltage standing wave ratio (VSWR) measurements were performed to study antenna transmission in snow. In all cases, the VSWR of the transmitting and receiving antennas demonstrates good transmission and reception when buried in the surface snow (Gerhardt and others, 2010; Barrella and others, 2011). Noise above and below the receiver bandwidth was filtered with MiniCircuits NHP and NLP filters, and amplified by a $62.4 \mathrm{~dB}$ Miteq AM-1660 lownoise amplifier (typical noise figure of $1.5 \mathrm{~dB}$ ). Signals were attenuated by $3-20 \mathrm{~dB}$ where appropriate, to remain in the linear regime of the amplifier.

For the 2006 season (Barrella and others, 2011), the transmitter and receiver were Seavey radio horns used in the ANITA (ANtarctic Impulsive Transient Antenna) experiment (ANITA Collaboration, 2009), with a bandwidth of [2001300] MHz. In the data from the 2010 season, the receiver and transmitter were log-periodic dipole arrays (LPDAs; 
Create Corp. CLP5130-2N) with a bandwidth of [1001300] $\mathrm{MHz}$. The Seavey is a dual polarization quad-ridge horn antenna that has higher gain above $200 \mathrm{MHz}$ than the LPDA. The LPDA antennas have a wider bandwidth, but stretch the signal in time with respect to the horn (Barwick and others, 2015). In the 2011 season, the data were recorded with a Seavey transmitter and an LPDA receiver. The 2010 data have been published (Hanson, 2011, 2013). In this work, the thickness results from 2010 are compared to three new measurements, and a new reflection coefficient and attenuation length analysis are presented.

In the surface test, we measured the pulse propagation time over the $543 \pm 7 \mathrm{~m}$ baseline, and extracted the surface index of refraction from the speed. The result was $n_{\text {surf }}=1.29 \pm 0.02$, and is needed for the boundary conditions in the shelf-thickness model. In the vertical bounce measurements, where the transmitter and receiver are colocated, the separation in 2006 was typically 9 m. In 2010 and 2011 the separation was 19 and $23 \mathrm{~m}$, respectively. This ensures that the receiver is in the far field of the transmitter during calibration. Comparing vertical bounce soundings to calibration measurements allows derivation of $\langle L\rangle$ assuming a value for $\sqrt{R}$.

The angled-bounce measurements are also compared to calibration measurements and vertical bounce cases to measure both $\langle L\rangle$ and $\sqrt{R}$. Angled signals were captured without having to account for complex ray tracing near the surface. During angled bounce tests, the transmitter and receiver were angled $45^{\circ}$ downward from horizontal. For the angled bounce measurements, the 2010 baseline was $977 \pm 7 \mathrm{~m}$, and the 2011 baseline was $543 \pm 7 \mathrm{~m}$. The angled bounce measurements in 2010 and 2011 had signal path lengths of $1517 \pm 8 \mathrm{~m}$ and $1272 \pm 7 \mathrm{~m}$, respectively. The incident angle with respect to normal refracts closer to $30^{\circ}$ when the pulse reaches the ocean, because the firn index $n_{\text {firn }}=1.3$ is smaller than the bulk ice index $n_{\text {ice }}=1.78$.

The Friis equation relates the power received, $P_{\mathrm{r}}$, to the transmitted power $P_{\mathrm{t}}$ in a lossless medium at a given wavelength. For two identical antennas in air, it may be written

$$
P_{\mathrm{r}}=\frac{P_{\mathrm{t}}\left(G_{\mathrm{a}} C\right)^{2}}{(4 \pi \nu d)^{2}}=\frac{P_{0}}{d^{2}}
$$

Here $G_{a}$ is the intrinsic gain of the antennas and $\nu$ is the frequency. $P_{\mathrm{r}}$ and $P_{\mathrm{t}}$ are the received and transmitted power, respectively. To account for absorption losses and possible losses upon reflection, the Friis equation is modified to

$$
P_{\mathrm{r}}=\frac{P_{0} R G_{1} G_{2}}{d^{2}} \exp \left(-\frac{2 d}{\langle L\rangle}\right)
$$

By convention, the factor of 2 in the exponential means $\langle L\rangle$ refers to electric field, and the reflection coefficient for the power is $R$. The factor $G_{1} G_{2}$ accounts for the relative power radiation pattern of the transmitter and receiver (Table 1). $G_{1}$ and $G_{2}$ are 1 for the vertical bounce measurements, in which the signal is transmitted and received in the forward direction of the antennas. As the angle at which the signal interacts with the antenna increases, $G_{1} G_{2}$ decreases from 1 according to the antenna radiation patterns. The radiation patterns have been both simulated and measured (ANITA Collaboration, 2009; Barwick and others, 2015). Manipulating Eqn (12) gives Eqn (13), the left-hand side of which may be plotted vs path length $d$ to obtain a line with a slope $-1 /\langle L\rangle$, and a constant $y$-intercept. The reflection coefficient is treated as a free parameter in the fit. The error in the
Table 2. A summary of total and physical time delays for the various seasons, and calculated shelf thicknesses. The physical time delay $\Delta t_{\text {phys }}$ is the measured delay $\Delta t_{\text {meas, }}$ with equipment delays subtracted. The total precision is quoted in the earlier measurements (Gerhardt and others, 2010; Barrella and others, 2011). The width of the reflected pulse, $\sigma_{\text {pulse, }}$ is caused by the response of the antennas

\begin{tabular}{cccccccc}
\hline Year & $\Delta t_{\text {meas }}$ & $\Delta t_{\text {phys }}$ & $\sigma_{\text {stat }}$ & $\sigma_{\text {sys }}$ & $\sigma_{\text {pulse }}$ & $\sigma_{\text {tot }}$ & $d_{\text {ice }}$ \\
& $\mathrm{ns}$ & $\mathrm{ns}$ & & & & & $\mathrm{m}$ \\
& & & & & & & \\
\hline 2006 & - & 6783 & - & - & - & 10 & $577.5 \pm 10$ \\
2009 & - & 6745 & - & - & - & 15 & $572 \pm 6$ \\
2010 & 7060 & 6772 & 5.0 & 8.0 & 10 & 14 & $576 \pm 6$ \\
2011 & 6964 & 6816 & 4.0 & 5.0 & 10 & 12 & $580 \pm 6$ \\
& & & & & & & \\
\hline
\end{tabular}

left-hand side of Eqn (13) is given by Eqn (14).

$$
\begin{aligned}
f(d) & =\ln \sqrt{\frac{d^{2} P_{\mathrm{r}}}{R G_{1} G_{2}}}=-d /\langle L\rangle+\text { const } \\
\sigma_{f} & =\sqrt{\left(\frac{\sigma_{d}}{d}\right)^{2}+\left(\frac{\sigma_{P, r}}{2 P_{\mathrm{r}}}\right)^{2}}
\end{aligned}
$$

\section{ICE-THICKNESS RESULTS}

The measured propagation times, both total and corrected for cable delays, are shown in Table 2 , along with statistical and systematic uncertainties. Using Eqns (8-10), the times are converted to thickness. Systematic errors arise from cables, the response time of the pulser, and delay generator precision (see Fig. 2). For the long cables, a conservative 5\% error in the propagation time is assumed, because the endpoints of the baselines were measured with GPS, with waypoint precision of $5 \mathrm{~m}$. The distance between the 2006 site and the site for the remaining three thickness measurements was $\sim 1 \mathrm{~km}$, and the GPS location of the latter site is accurate to within a horizontal uncertainty of $5 \mathrm{~m}$.

The reflected pulse from the 2009 set-up was only several $\mathrm{mV}$ above noise backgrounds, so the entire pulse width was included as systematic error (Gerhardt and others, 2010). For the 2011 data, the location and uncertainty in peak voltage oscillations in the reflections were used instead, because the signal was well above backgrounds. The low-frequency ringing in the reflected data originates from group delay in the LPDA, which is $10 \mathrm{~ns}$ at $0.2 \mathrm{GHz}$ (the lowest frequency emitted by the transmitter). When folded into the timing uncertainties, smaller but comparable errors to 2006 are obtained for the thickness. Timing uncertainties are lowest in 2006 because both transmitter and receiver were Seavey horns, which have lower group delay than the LPDA.

In general, statistical errors come from Eqn (10), with $n=1.78 \pm 0.02$, and total timing error from Table 2 . The magnitude of $\sigma_{n}$ comes from measurements made at the surface ( $n_{\text {surf }}=1.29 \pm 0.02$ ) (Hanson, 2013). Fluctuations in $n$ are largest at the surface, making this a conservative estimate for the bulk ice, and it is similar to previous work (Gerhardt and others, 2010; Barrella and others, 2011). The total error from 2006 is higher because a larger error on the dielectric constant was used. The mean thickness over all seasons is $d_{\text {ice }}=576 \pm 8 \mathrm{~m}$ (statistical and systematic added in quadrature). Errors in Table 2 other than from the index of refraction are treated as systematic. A linear fit to the four 

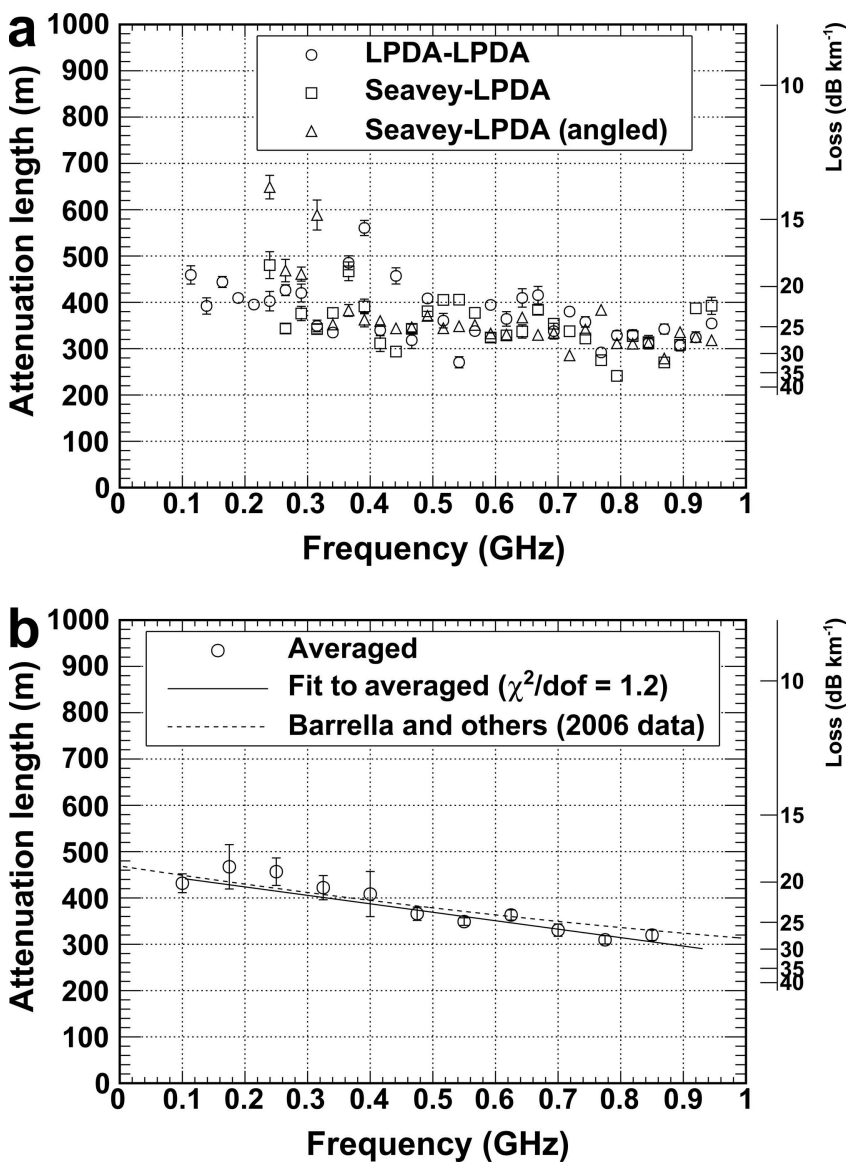

Fig. 4. (a) The depth-averaged attenuation length vs frequency, with standard deviations from error propagation in Eqn (18). The attenuation length is converted to englacial loss $\left(\mathrm{dB} \mathrm{km}^{-1}\right)$, at right. (b) The data from (a) averaged into $75 \mathrm{MHz}$ bins, with standard deviations. The linear fit has $\chi^{2} /$ dof $=1.2$, a slope of $-180 \pm$ $40 \mathrm{~m} \mathrm{GHz}^{-1}$ and an offset of $460 \pm 20 \mathrm{~m}$. The dashed line is a fit to prior data taken $1 \mathrm{~km}$ from our site (Barrella and others, 2011).

data points together yields a slope consistent with zero (within errors). The measurement from 2006 took place $1 \mathrm{~km}$ from the location of subsequent seasons, and does not deviate strongly from the mean.

\section{AVERAGE ATTENUATION LENGTH, $\langle L\rangle$, VS FREQUENCY}

The technique of measuring $\sqrt{R}$ and $\langle L\rangle$ simultaneously is more challenging than assuming a constant $\sqrt{R}$ and comparing the raw power spectra of only vertical bounce data and calibration data. Assuming a uniform reflection coefficient with respect to frequency assumes that the reflecting surface is dominated by specular, rather than diffuse, reflection. As long as the first few Fresnel zones $D_{m}$ of the transmitted pulse are not significantly larger than the horizontal correlation length $L_{C}$ of roughness features along the shelf base, then the effect of the vertical roughness scales on the reflection coefficient is avoided (Peters and others, 2005). Prior data collected at two locations on the Ross Ice Shelf, near Moore's Bay, indicate horizontal correlation lengths $L_{C}=12.5 \mathrm{~m}$ and $L_{C}=27.5 \mathrm{~m}$ at the two sites (Neal, 1982). The glaciological Fresnel zone equation, for an observation point a distance $h$ above the snow surface, with a shelf thickness of $z$, shelf index of refraction $n$, Fresnel zone number $m$, and an in-air wavelength $\lambda$ is

$$
D_{m} \approx \sqrt{2 m \lambda\left(h+\frac{z}{n}\right)}
$$

The approximation arises from the small angle approximation, and is sound because the Fresnel zones are small compared to $z$. The measurements take place at the surface, so $h=0$. Using $n=1.78, \lambda=3 \mathrm{~m}$ and the measured shelf thickness, Eqn (15), gives $D_{1}=10-40 \mathrm{~m}$, for the bandwidth. Vertical rms fluctuations at the ocean/ice surface were reported to be be $3 \mathrm{~cm}$ and $10 \mathrm{~cm}$ for two sites, spread out over a typical length scale of $L_{C}$. Vertical height fluctuations of $10 \mathrm{~cm}$ and $3 \mathrm{~cm}$ spread out over $12.5 \mathrm{~m}$ and $27.5 \mathrm{~m}$, respectively, mean that specular reflection is a good approximation for this bandwidth (Neal, 1982). The attenuation lengths derived assuming constant $\sqrt{R}$ are revised in the next section, to account for reflection loss $(\sqrt{R}<1.0)$.

Consider the calibration pulse, $V_{C}$, the vertical bounce pulse, $V_{\text {ice, }}$ and the depth-averaged attenuation length vs frequency, $\langle L(\nu)\rangle$, all at a frequency $\nu$ :

$$
\begin{aligned}
V_{\mathrm{C}}(\nu) & =V_{0} / d_{\mathrm{C}} \\
V_{\text {ice }}(\nu) & =\frac{V_{0}}{d_{\text {ice }}} \exp \left(-\frac{d_{\text {ice }}}{\langle L(\nu)\rangle}\right) \\
\langle L(\nu)\rangle & =\frac{d_{\text {ice }}}{\ln \left(\left(V_{\mathrm{C}}(\nu) d_{\mathrm{C}}\right) /\left(V_{\text {ice }}(\nu) d_{\text {ice }}\right)\right)}
\end{aligned}
$$

Because the surface of the firn is snow, with a density of $0.4 \mathrm{~g} \mathrm{~cm}^{-3}$ and an index of refraction $n=1.3$, the reflection coefficient (for power) between air and snow is $\sim 0.02$, so potential interference from surface reflections is not expected to modify Eqn (16). The antennas were placed at the maximum height allowed by the cables and other equipment $(1.5 \mathrm{~m})$, and this calibration was compared to the case with the antennas buried in snow slots. Because of the low snow density, dielectric absorption is negligible over the calibration distances $(23 \mathrm{~m})$. The antenna calibrations produced similar waveforms with the antennas lowered in snow. The waveform amplitude increases when LPDAs are in the snow, due to the shift in the lower cut-off frequency by the index of refraction. This effect is confirmed in Numerical Electromagnetic Code (NEC) antenna simulations, and VSWR data (Barwick and others, 2015).

The 2011 data are shown in Figure 4a. In Eqns (16-18), the voltages are defined $V \propto \sqrt{P(\nu)}$, where $P$ is the measured power at the frequency $\nu$. The antenna impedance is the same for the calibration and bounce studies, making it irrelevant in the ratio in Eqn (18) (Barwick and others, 2015). The 2011 power spectra begin at the lowfrequency cut-offs of the transmitter type $(200 \mathrm{MHz}$ for the Seavey, and $100 \mathrm{MHz}$ for the LPDA). The englacial loss $\left(\mathrm{dB} \mathrm{km}^{-1}\right)$ is also shown (Eqn (3)).

The 2011 data extend to $0.850 \mathrm{GHz}$, where the signal-tonoise ratio is close to 1.0 , and the error bars are the standard deviation from error propagation in Eqn (18). About $10 \mathrm{~m}$ of the error is due to uncertainty in the shelf thickness, and $10 \mathrm{~m}$ is due to uncertainty in the power spectrum. Data above $0.850 \mathrm{GHz}$ appear to be rising due to noise floor contributions. Also, systematic fluctuations in the vertical bounce power spectra lead to systematic fluctuations in $\langle L(\nu)\rangle$. Systematic errors arise from differences in system frequency response after changing the transmitter location and type, and reflections within the system. The angled bounce data at 0.240 and $0.315 \mathrm{GHz}$, in particular, are 

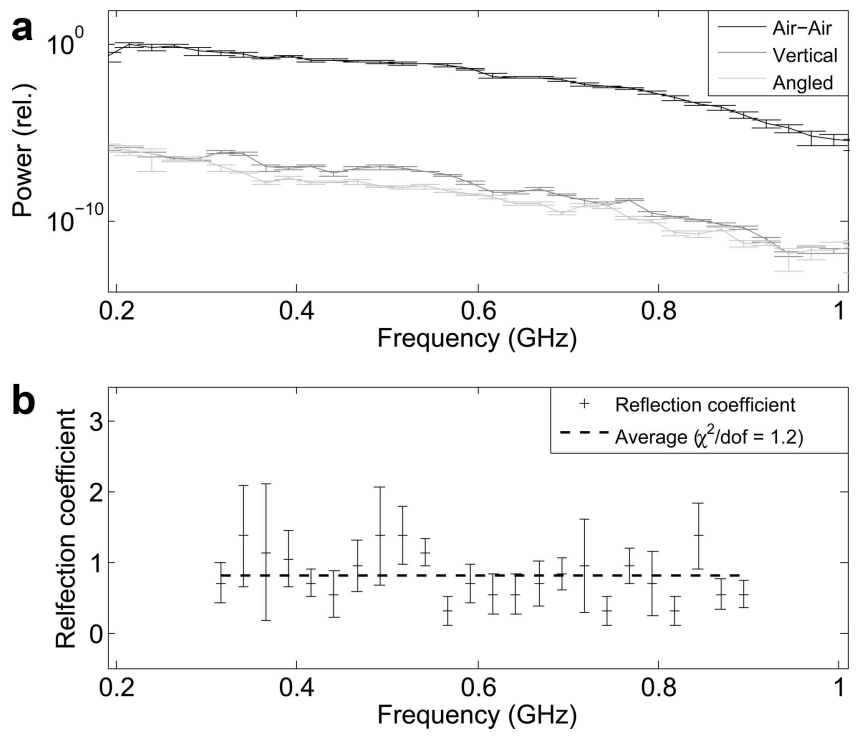

Fig. 5. The electric-field reflection coefficient, $\sqrt{R}$, vs frequency. The three power spectra correspond to three measurements: a surface power calibration (black), vertical bounce (dark grey) and angled bounce (light grey) cases. The three measurements at each frequency determine a reflection coefficient through a linear fit to Eqn (13), with errors from Eqn (14) attributed to $\sqrt{R}$.

systematically high. The Seavey transmitter was placed in a snow cavity rather than fully buried for the angled test, which can lead to cavity resonance effects.

The frequency resolution is maximized in Figure $4 a$, with no window function. A higher resolution extends the upper frequency limit by avoiding folding noise into the highestfrequency bins. The correction for potential angular dependence of the reflection coefficient only applies to the angled bounce data $(\sim 4 \mathrm{~m})$. In Figure $4 \mathrm{a}$, the data are averaged into $0.075 \mathrm{GHz}$ bins, with a linear fit. The best-fit slope is $-180 \pm 40 \mathrm{~m} \mathrm{GHz}^{-1}$, and the best-fit offset is $460 \pm 20 \mathrm{~m}$ $\left(95 \%\right.$ confidence level, $\chi^{2} /$ dof $\left.=1.2\right)$. Data above $0.850 \mathrm{GHz}$ are neglected in the average and fit shown in Figure 4; however, the $\chi^{2} /$ dof only increases to 1.8 if it is included. As in Figure 4a, the averaged attenuation length is converted to $\mathrm{dB} \mathrm{km}^{-1}$ on the right-hand $y$-axis using Eqn (3).

Despite the systematic fluctuations, the fit to the data in Figure 4 is in close agreement with the quadratic fit to the data from 2006 (Barrella and others, 2011). In the publication of the 2006 data, the reflection loss was assumed to be $0 \mathrm{~dB}$. If a lower value is assumed (see below), the attenuation length increases, because the returned voltage per unit frequency in Eqn (18) must remain constant. The level of systematic variation in $\sqrt{R}$ shown below will also generate $\sim 5 \%$ systematic uncertainty in $\langle L\rangle$, but only to increase it. The 2006 and 2011 data agree, even though the measurements were made $1 \mathrm{~km}$ apart. The area of Moore's Bay near Minna Bluff is far from any zones of high glacial velocity that could cause depth or basal reflection variations, and crevasses have not been observed in the area, so the ice is expected to be relatively uniform.

\section{BASAL REFLECTION COEFFICIENT}

The 2006 season $\langle L\rangle$ results were derived from vertical bounce measurements assuming $\sqrt{R}=1.0$. Using the path lengths derived from shelf thickness, and the measured
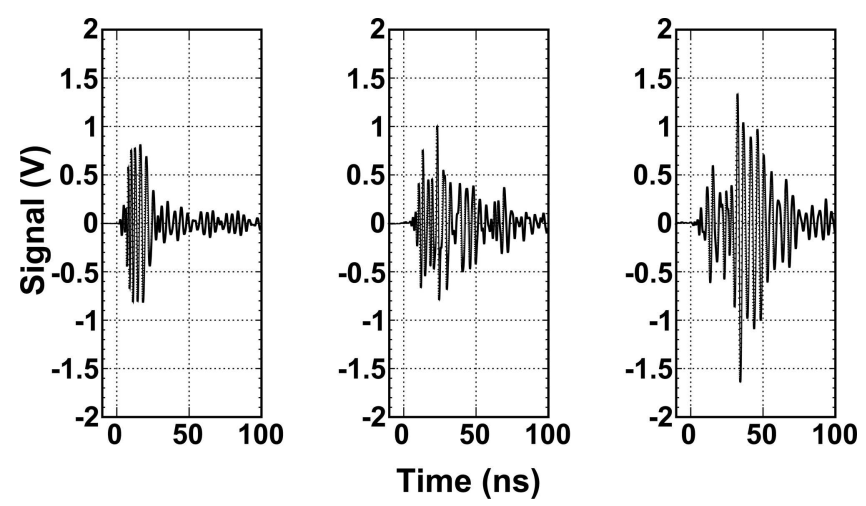

Fig. 6. Typical waveforms from 2011, averaged over 100 triggers. (a) The calibration pulse. (b) The vertical bounce reflection. (c) The angled bounce reflection. The vertical and angled bounce data have been amplified by the $60 \mathrm{~dB}$ Miteq amplifier. The data have been scaled to account for in-line attenuators (the data were kept within the amplifier linear range).

power spectra of the calibration, vertical bounce and angled bounce reflections, $\sqrt{R}$ can be derived separately from the attenuation length. The errors in $\sqrt{R}$ arise from propagating errors in path length (from thickness, and geometry) and returned power through Eqn (13).

The three tests (calibration, vertical and angled bounce) serve as three measurements of $f(d)$ for different values of the path length $d$, given the free parameter $\sqrt{R}$. The measurements are compared to the linear model $f_{\text {model }}=-d /\langle L\rangle+f_{0}$, which is scanned through $(\sqrt{R},\langle L\rangle)$ parameter space. The $y$-intercept is irrelevant to the physics, coming from the linear fit upon each iteration. (The overall power at a given frequency is relative to the calibration pulse power.) Each iteration produces a $\chi^{2}$ value, and $(\sqrt{R},\langle L\rangle)$ were scanned until a global minimum was reached at each frequency.

The averaged power spectra of the time-dependent waveforms are shown in Figure $5 \mathrm{a}$. The spectra are constructed from averaging the modulus-squared of the fast Fourier transform of the time-dependent signals, and plotted relative to the maximum calibration power. The error bars are the error in the mean for each bin. Examples of waveforms from which these power spectra are derived are shown separately in Figure 6. For all recorded waveforms, a sampling rate of $5 \mathrm{GHz}$ was used on the $1 \mathrm{GHz}$ bandwidth oscilloscope. The spectra in Figure 5 have a frequency resolution of $0.025 \mathrm{GHz}$.

The 2010 data for $\sqrt{R}$ vs $\langle L\rangle$ have been analysed by Hanson (2011, 2013). The basic results were $480 \mathrm{~m}$ $\leq\langle L\rangle \leq 510 \mathrm{~m}\left(17-18 \mathrm{~dB} \mathrm{~km}^{-1}\right)$, and $0.72 \leq \sqrt{R} \leq 0.88$ $(1.1-2.8 \mathrm{~dB}$ loss), for the average attenuation length and reflection coefficient (68\% confidence level). The set-up (Fig. 2) demonstrated good transmission through surface snow for frequencies below $0.180 \mathrm{GHz}$ that season, and the LDPA lower limit in the snow is $0.080 \mathrm{MHz}$. The index of refraction of snow extends the LPDA response to $0.080 \mathrm{GHz}$ from a lower limit of $0.105 \mathrm{GHz}$ (Barwick and others, 2015). A shorter angled bounce baseline $(543 \pm 7 \mathrm{~m})$ was chosen for the 2011 season, relative to the prior year, to boost signal at higher frequencies; however, the snow absorption effect was not observed in 2011.

Figure 5b shows the $\sqrt{R}$ results from the 2011 season. The baseline sets the path length difference between the 
Table 3. Summary of dielectric parameters. The first column is the frequency, $\nu$, followed by the attenuation lengths, which are uncorrected $\left(\left\langle L_{0}\right\rangle\right)$ and corrected $(\langle L\rangle)$ for $\sqrt{R}=0.82 \pm 0.07$. The fourth column is $\langle L\rangle$ expressed in $\mathrm{dB} \mathrm{km}^{-1}$. The imaginary part of the dielectric constant, $\epsilon^{\prime \prime}$, is shown in the fifth column. The final column shows $\nu \tan \delta(\mathrm{GHz})$. The typical error on the quantity $\nu \tan \delta$ is $0.2 \times 10^{-4}$

\begin{tabular}{lccccc}
\hline$\nu$ & $\left\langle L_{0}\right\rangle$ & $\langle L\rangle$ & $\begin{array}{c}\langle l\rangle \\
\mathrm{dB} \mathrm{km}{ }^{-1}\end{array}$ & $\epsilon^{\prime \prime} \times 10^{3}$ & $\begin{array}{c}\nu \tan \delta \times 10^{4} \\
\mathrm{GHz}\end{array}$ \\
$\mathrm{GHz}$ & $\mathrm{m}$ & $\mathrm{m}$ & & & \\
\hline 0.100 & 432 & 449 & 19.3 & 3.8 & 1.2 \\
0.175 & 467 & 487 & 17.8 & 2.0 & 1.1 \\
0.250 & 457 & 476 & 18.2 & 1.4 & 1.1 \\
0.325 & 422 & 438 & 19.8 & 1.2 & 1.2 \\
0.400 & 408 & 423 & 20.5 & 1.0 & 1.3 \\
0.475 & 366 & 378 & 23.0 & 0.95 & 1.4 \\
0.550 & 349 & 360 & 24.1 & 0.86 & 1.5 \\
0.625 & 363 & 375 & 23.2 & 0.72 & 1.4 \\
0.700 & 331 & 341 & 25.5 & 0.71 & 1.6 \\
0.775 & 310 & 319 & 27.2 & 0.69 & 1.7 \\
0.850 & 320 & 329 & 26.4 & 0.61 & 1.6 \\
Ave. & $380 \pm 16$ & $400 \pm 18$ & $22 \pm 1$ & $1.3 \pm 0.3$ & $1.37 \pm 0.06$ \\
\hline
\end{tabular}

angled and vertical cases, introducing a trade-off. A shorter baseline causes the attenuation length to become large compared to the difference in path length between the angled and vertical bounce tests $(\sim 130 \mathrm{~m}$ in 2011). At low frequencies, the difference in power loss between vertical and angled cases becomes smaller than the errors in the power spectra $(\sim 3 \mathrm{~dB}$ at $0.300 \mathrm{GHz})$. Alternatively, the baseline for the angled bounce can be increased, which increases the path length difference between angled and vertical bounces. While this increases the low-frequency precision, the high-frequency precision suffers due to increased absoprtion in the angled bounce data. The vertical and angled signal power are equal within statistical errors up to $0.300 \mathrm{GHz}$ in Figure $5 \mathrm{a}$, but differences in the vertical and angled power are measurable up to $0.850 \mathrm{GHz}$.

Figure $5 \mathrm{~b}$ shows $\sqrt{R}$, with statistical errors from the fit, at each frequency. The errors are conservative, in that all the deviation from a perfect linear model (Eqn (14)) is attributed to error in $\sqrt{R}$. The mean is $\sqrt{R}=0.82 \pm 0.07(-1.7 \mathrm{~dB})$, and a flat model at this mean has a chi-squared result of $\chi^{2} /$ dof $=1.2$. Despite fluctuations in the data, no data point deviates above the physical region by much more than one standard deviation. At each point in the bandwidth where the gap between the reflected and calibration spectra randomly decreases, the parameter $\sqrt{R}$ must fluctuate upwards to produce a linear fit to $f(d)$. These data have larger error bars, because the deviation in $f(d)$ from a linear model is larger for those bins. An unweighted flat-line fit to the data is shown; this fit produces the same results, within statistical uncertainties, as a weighted fit (that de-emphasizes the points with large errors). If the bins with $\sqrt{R}>1$ are ignored in the fit, then the mean decreases by $20 \%(\sqrt{R}=0.6)$.

Ignoring the data above 1.0, however, would raise the attenuation length results, because the total power loss must be conserved (Table 3). As the attenuation length has the stronger impact on the design of the future ARIANNA detector, relative to the reflection coefficient, it is important to be conservative with regard to the attenuation length in
Table 3 (Dookayka, 2011). The origin of the roughness in the reflected spectra is likely noise interference, since the signal-to-noise ratio is lower than in the calibration study.

Finally, knowledge of the basal reflection coefficient allows the correction of the attenuation length numbers in Figure 4 to more realistic values. If $\left\langle L_{0}\right\rangle$ is the measured attenuation length, assuming $\sqrt{R}=1.0$, then the actual attenuation length $\langle L\rangle$ can be expressed as

$$
\frac{\langle L\rangle}{\left\langle L_{0}\right\rangle}=\left(1+\frac{\left\langle L_{0}\right\rangle}{2 d_{\text {ice }}} \ln R\right)^{-1}
$$

Using the $\left\langle L_{0}\right\rangle$ values from Figure 4 , Table 3 shows the $\langle L\rangle$ results for the mean value of $\sqrt{R}=0.82 \pm 0.07$, vs frequency. Table 3 also shows the imaginary part of the dielectric constant, derived from $n^{\prime \prime}$, via $\langle L\rangle^{-1}=n^{\prime \prime} k$, where $k$ is the free-space wavenumber. Assuming $\tan \delta \ll 1$, the expression $\epsilon^{\prime \prime}=2 n^{\prime \prime} \sqrt{\epsilon^{\prime}}$, with $\sqrt{\epsilon^{\prime}}=1.78$, relates the two quantities.

The $\epsilon^{\prime \prime}$ results are in agreement with an earlier lowfrequency projection for typical ice-shelf temperatures (Matsuoka and others, 1996). The Debye model predicts $\epsilon^{\prime \prime} \propto \nu^{-1}$ for frequencies below $2 \mathrm{GHz}$, and the $\epsilon^{\prime \prime}$ data follow this trend. The quantity $\nu \tan \delta$ is expected to be small and constant for a simple dielectric material, and Table 3 also displays this quantity in the final column, which agrees with an estimate from analysis of the 2006 data (Barrella and others, 2011). Although $\nu \tan \delta$ varies with frequency, this variation is such that no measurement is more than one standard deviation $\left(0.2 \times 10^{-4}\right)$ from the mean $(1.37 \pm 0.06)$.

\section{POLARIZATION MEASUREMENTS}

The $\sqrt{R}$ result shows that little power is lost from the basal reflection. In this section, we assess potential losses by scattering or rotation of the linearly polarized signal. For any non-ideal linearly polarized antenna system, a small amount of power can leak into the cross-polarized channel. Significant transfer of power into the cross-polarized direction would indicate polarization rotation in the ice, and bias the attenuation length results. Birefringence and surface roughness effects at the water-ice interface at the bottom of the ice shelf are expected to generate power in the cross-polarized direction.

To quantify the polarization rotation, the cross-polarization fraction, $F_{\text {ice, }}$ was measured in the vertical bounce configuration, and compared to $F_{\text {air }} F$ is defined in Eqn (20), where $P_{\perp}$ and $P_{\|}$refer to the measured power in the crosspolarized and co-polarized direction with respect to the linear polarization of the transmitter at a given frequency:

$$
\digamma=\frac{P_{\perp}}{P_{\perp}+P_{\|}}
$$

The leakage between co-polarized and cross-polarized channels is expected to be low across the bandwidth, but difficult to observe at high frequencies. Cross-polarized signals are weaker than co-polarized, and the vertical bounce data in the cross-polarized state are subject to noise interference above $0.4 \mathrm{GHz}$. The intrinsic transfer into the cross-polarized direction of a specified antenna pair was estimated by facing the transmitter toward the receiver in air. $F_{\text {air }}$ is computed from the power observed between copolarized and cross-polarized orientation of the receiver. The results of this study are shown in the third column of 
Table 4. It was verified that the snow surface $1.5 \mathrm{~m}$ below the antennas scatters back a negligible amount of power.

$F_{\text {ice }}$ was obtained from the vertical bounce set-up, with a Seavey transmitter and LPDA receiver. The Seavey antenna transmits very little power below $0.175 \mathrm{GHz}$, and the crosspolarized signal is weaker than the co-polarized signal, limiting $F_{\text {ice }}$ results to frequencies below $0.4 \mathrm{GHz}$. These measurements are shown in column 4 of Table 4 . These data can be compared to measurements taken in 2010, in which $F_{\text {ice }}$ and $F_{\text {air }}$ were shown to agree at $0.1 \mathrm{GHz}$ with a LPDA transmitter and LPDA receiver at the same location as the 2011 measurements (Hanson, 2011). A comparison of $F_{\text {air }}$ with $F_{\text {ice }}$ reveals no excess power in the cross-polarization direction, with the possible exception of data at $0.400 \mathrm{GHz}$, which show a $2 \sigma$ deviation from intrinsic antenna effects. These data do not confirm the $F_{\text {ice }}$ analysis of the 2006 data, which showed $F_{\text {ice }}=0.7$ at $0.4 \mathrm{GHz}$.

\section{DISCUSSION}

The data are in agreement with independent analyses and models. A study from Greenland found the total transfer function of the Greenland ice sheet, and models the different contributions from basal reflection and attenuation (Paden and others, 2005). A reflection coefficient (for power) of $-37 \mathrm{~dB}$ is reported for the North Greenland Ice Core 2 (NGRIP2) location, and ice absorption of $\sim 56 \mathrm{~dB}$. Given the depth of $3.1 \mathrm{~km}$, a loss rate of $\sim 9.0 \mathrm{~dB} \mathrm{~km}^{-1}$ is obtained. (The Greenland study was limited to 0.11$0.5 \mathrm{GHz}$.) The upper half of the Greenland ice sheet is colder than Moore's Bay, lowering the attenuation rate through temperature dependence of $\epsilon^{\prime \prime}$. The reflection coefficient from that study $(-37 \mathrm{~dB})$ is much smaller than that of Moore's Bay. However, other authors have estimated it to be higher (Bamber and others, 2001; Avva and others, 2014), with an absorption rate of $9.2 \mathrm{~dB} \mathrm{~km}^{-1}$, conservatively assuming no reflection loss (attributing all loss to absorption). The Greenland site also exhibits a frequency dependence that produces a change of $8.5 \mathrm{~dB} \mathrm{~km}^{-1}$ over the bandwidth $(0.11-0.5 \mathrm{GHz})$. The slope of the loss rate vs frequency is therefore $8.5 /(0.55-0.11) \approx 22 \mathrm{~dB} \mathrm{~km}^{-1}$ $\mathrm{GHz}^{-1}$. The corresponding value for the ARIANNA site is $9.3 \mathrm{~dB} \mathrm{~km}^{-1} \mathrm{GHz}^{-1}$, from Table 3 .

Another study presents models for ice absorption across the entire Antarctic continent, given an array of inputs, such as temperature and chemistry data (Matsuoka and others, 2012). That expansive study presents results for shelf and sheet depth across the continent, and the portion depicting the Ross Ice Shelf, near the ARIANNA site, is in agreement with our thickness measurements. The RIS depth is peaked at $500 \mathrm{~m}$ in that model, and we find $576 \pm 8 \mathrm{~m}$. The inputs to this model indicate that the Ronne Ice Shelf has smaller absorption rates $\left(\mathrm{dB} \mathrm{km}^{-1}\right)$ than the Ross Ice Shelf, which leads to a double-peaked distribution of loss rates, with one peak near $12.5 \mathrm{~dB} \mathrm{~km}^{-1}$, and the other near $22.5 \mathrm{~dB} \mathrm{~km}^{-1}$. The ARIANNA site average absorption rate is within one standard deviation of the mean for the entire distribution $\left(15.1 \pm 6.2 \mathrm{~dB} \mathrm{~km}^{-1}\right)$, and is in agreement with the second peak in the distribution of loss rates, corresponding to the Ross Ice Shelf.

Finally, a study of the Ross Ice Shelf at $2 \mathrm{MHz}$ reveals large-scale thickness uniformity in the shelf, up to $40 \mathrm{~km}$ from the grounding line of the glaciers flowing into the shelf
Table 4. A comparison of cross-polarization fraction measurements vs frequency

\begin{tabular}{lcc}
\hline Frequency & $F_{\text {air }}$ & $F_{\text {ice }}$ \\
$\mathrm{GHz}$ & & \\
\hline 0.175 & $0.06 \pm 0.02$ & $0.08 \pm 0.05$ \\
0.200 & $0.04 \pm 0.01$ & $0.01 \pm 0.01$ \\
0.225 & $0.04 \pm 0.02$ & $0.02 \pm 0.01$ \\
0.250 & $0.02 \pm 0.01$ & $0.01 \pm 0.01$ \\
0.275 & $0.02 \pm 0.01$ & $0.02 \pm 0.01$ \\
0.300 & $0.02 \pm 0.01$ & $0.07 \pm 0.04$ \\
0.325 & $0.01 \pm 0.005$ & $0.03 \pm 0.01$ \\
0.350 & $0.04 \pm 0.01$ & $0.08 \pm 0.03$ \\
0.375 & $0.02 \pm 0.01$ & $0.11 \pm 0.07$ \\
0.400 & $0.03 \pm 0.01$ & $0.22 \pm 0.09$ \\
\hline
\end{tabular}

(MacGregor, 2011). The measurements are obtained from basal echoes with travelling transmitters and receivers at the surface. In some cases, multiple echoes are observed, corresponding to multiple round trips made by the signal, from surface to base. This technique provides excellent constraints on the thickness and absorption rate. Specifically, this study shows that our depth measurement is typical for large expanses of ice, a key requirement for large-scale ground arrays in neutrino detectors.

\section{CONCLUSION}

During the 2011/12 Antarctic season, radio-echo sounding measurements were performed in Moore's Bay with highvoltage broadband RF pulses in the $0.1-0.850 \mathrm{GHz}$ bandwidth, to understand the dielectric properties of the ice shelf. The shelf thickness determined from the total propagation time was $576 \pm 8 \mathrm{~m}$. The echo soundings revealed depth-averaged attenuation lengths well fit by the linear function $\langle L(\nu)\rangle=(460 \pm 20)-(180 \pm 40) \times \nu \mathrm{m}$ $\left(19.3-26.4 \mathrm{~dB} \mathrm{~km}^{-1}\right)$, where $\nu$ is the frequency $(\mathrm{GHz})$. The $\chi^{2} /$ dof of this linear fit to the combination of multiple datasets was 1.2, with 9 degrees of freedom. The fit is consistent with prior measurements (Barrella and others, 2011), and the functional dependence is compatible with theoretical expectations (Matsuoka and others, 1996; Somaraju and others, 2006).

Vertical echo soundings were compared to echo soundings with a $543 \pm 7 \mathrm{~m}$ baseline between transmitter and receiver, which allowed independent measurement of the basal reflection coefficient, found to be $\sqrt{R}=0.82 \pm 0.07$ $(-1.7 \mathrm{~dB})$. The slope of $\sqrt{R}$ vs frequency is consistent with a flat-mirror approximation. The average value of $\sqrt{R}$ is consistent with earlier studies performed at lower frequencies (Neal, 1979). The short duration of the observed pulses $(90 \%$ of the power contained within $100 \mathrm{~ns})$ precludes significant multi-path effects. The Fresnel zones of the pulses at the shelf base are not significantly larger than measured horizontal correlation lengths. After correcting attenuation lengths for the effect of $\sqrt{R}$ on returned power, dielectric quantities like $\epsilon^{\prime \prime}$ and $\nu \tan \delta$ were derived. The results for $\epsilon^{\prime \prime}$ and $\nu \tan \delta$ agree with theoretical expectations (Matsuoka and others, 1996). Finally, the fraction of scattered power by the ice into the cross-polarized direction, $F_{\text {ice, }}$ is $<10 \%$ $(0.100-0.400 \mathrm{GHz})$, compatible with the fraction of power 
due to intrinsic limitations of the transmitting and receiving antennas. Both the large value of $\sqrt{R}$ and the small value of $F_{\text {ice }}$ suggest that the bottom surface of the Ross Ice Shelf at Moore's Bay is smooth. The measurements of $F_{\text {ice }}$ do not demonstrate any significant features below $0.400 \mathrm{GHz}$, where cross-polarized power is noise-limited. This result, combined with the measured field attenuation length at frequencies between 0.100 and $0.850 \mathrm{GHz}$, suggests that the Moore's Bay region of the Ross Ice Shelf will be an excellent medium for the ARIANNA high-energy neutrino project.

\section{ACKNOWLEDGEMENTS}

We thank the staff of Antarctic Support Contractors, Lockheed, Raytheon Polar Services, and the entire crew at McMurdo station for excellent logistical support. This work was supported by generous funding from the Office of Polar Programs and Physics Division of the US National Science Foundation, grant awards ANT-08339133, NSF-0970175 and NSF-1126672. In 2010, additional funding was provided through the US Department of Energy under contract DE-AC-76SF-00098. Finally, we thank David Saltzberg for comments and suggestions throughout the expeditions and analysis.

\section{REFERENCES}

Allison P and 47 others (2012) Design and initial performance of the Askaryan Radio Array prototype EeV neutrino detector at the South Pole. Astropart. Phys., 35, 457-477

AMANDA Collaboration (2006) Optical properties of deep glacial ice at the South Pole. J. Geophys. Res., 111(D13), D13203 (doi: 10.1029/2005JD006687)

ANITA Collaboration (2009) The Antarctic Impulsive Transient Antenna ultra-high energy neutrino detector: design, performance, and sensitivity for 2006-2007 balloon flight. Astropart. Phys., 32(1), 10-41

ANITA Collaboration (2010) Observational constraints on the ultrahigh energy cosmic neutrino flux from the second flight of the ANITA experiment. Phys. Rev. D, 82(2), 022004 (doi: 10.1103/PhysRevD.82.022004)

Askaryan GA (1962) Excess charge of an electron-photon shower and its coherent radio emission. Sov. Phys. JETP, 14(2), 441-442

Avva J, Kovac JM, Miki C, Saltzberg D and Vieregg AG (2014) An in situ measurement of the radio-frequency attenuation in ice at Summit Station, Greenland. arXiv:1409.5413

Bamber JL, Layberry RL and Gogineni SP (2001) A new thickness and bed data set for the Greenland ice sheet: 1. Measurement, data reduction, and errors. J. Geophys. Res., 106(D24), 33 773-33 780

Barrella T, Barwick S and Saltzberg D (2011) Ross Ice Shelf (Antarctica) in situ radio-frequency attenuation. J. Glaciol., 57(201), 61-66

Barwick SW (2007) ARIANNA: a new concept for UHE neutrino detection. J. Phys. Conf. Ser., 60, 276-283 (doi: 10.1088/17426596/60/1/060)

Barwick SW and 14 others (2015) The time-domain response of the ARIANNA detector. Astropart. Phys. J., 62, 139-151 (doi: 10.1016/j.astropartphys.2014.09.002)

Besson D and 42 others (2008) In-situ radioglaciological measurements near Taylor Dome, Antarctica and implications for UHE neutrino astronomy. Astropart. Phys., 29(2), 130-157
Bogorodsky VV, Bentley CR and Gudmandsen PE (1985) Radioglaciology. Reidel Publishing Co., Dordrecht

Dookayka K (2011) Characterizing the search for ultra-high energy neutrinos with the ARIANNA Detector. (PhD thesis, University of California, Irvine)

Dowdeswell JA and Evans S (2004) Investigations of the form and flow of ice sheets and glaciers using radio-echo sounding. Rep. Progr. Phys., 67(10), 1821-1861

Fahnestock MA, Scambos TA, Bindschadler RA and Kvaran G (2000) A millennium of variable ice flow recorded by the Ross Ice Shelf, Antarctica. J. Glaciol., 46(155), 652-664

Fretwell P and 55 others (2013) Bedmap2: improved ice bed, surface and thickness datasets for Antarctica. Cryosphere, 7(1), 375-393 (doi: 10.5194/tc-7-375-2013)

Gerhardt L and 6 others (2010) A prototype station for ARIANNA: a detector for cosmic neutrinos. Nucl. Instrum. Meth. Phys. Res. A, 624(1), 85-91

Hanson JC (2011) Ross Ice Shelf thickness, radio-frequency attenuation and reflectivity: implications for the ARIANNA UHE neutrino detector. Proceedings of the 32 nd International Cosmic Ray Conference, 11-18 August, Beijing, China. Institute of High Energy Physics, Beijing

Hanson JC (2013) The performance and initial results of the ARIANNA prototype. (PhD thesis, University of California, Irvine)

IceCube Collaboration (2013) Evidence for high-energy extraterrestrial neutrinos at the IceCube detector. Science, 342(6161), 1242856 (doi: 10.1126/science.1242856)

Klein, SR (2012) Radiodetection of neutrinos. Nucl. Phys. Proc. Suppl., 229-232, 284-288

Kravchenko I and 8 others (2012) Updated results from the RICE experiment and future prospects for ultra-high energy neutrino detection at the south pole. Phys. Rev. Lett. D, 85, 062004

MacGregor J, Anandakrishnan S, Catania GA and Winebrenner DP (2011) The grounding zone of the Ross Ice Shelf, West Antarctica, from ice-penetrating radar. J. Glaciol., 57(205), 917-928

Matsuoka K, Fujita S and Mae S (1996) Effect of temperature on dielectric properties of ice in the range 5-39 GHz. J. Appl. Phys., 80(10), 5884-5890

Matsuoka K, MacGregor JA and Pattyn F (2012) Predicting radar attenuation within the Antarctic ice sheet. Earth Planet. Sci. Lett., 359-360, 173-183 (doi: 10.1016/J.EPSL.2012.10.018</A >)

Neal CS (1979) The dynamics of the Ross Ice Shelf revealed by radio echo-sounding. J. Glaciol., 24(90), 295-307

Neal CS (1982) Radio echo determination of basal roughness characteristics on the Ross Ice Shelf. Ann. Glaciol., 3, 216-221

Paden J, Allen CT, Gogineni S, Jezek KC, Dahl-Jensen D and Larsen LB (2005) Wideband measurements of ice sheet attenuation and basal scattering. Geosci. Remote Sens. Soc. Newsl., 2(2) (doi: 10.1109/LGRS.2004.842474)

Peters M, Blankenship DD and Morse DL (2005) Analysis techniques for coherent airborne radar sounding: application to West Antarctic ice streams. J. Geophys. Res., 110(B6), B06303 (doi: 10.1029/2004JB003222)

Rignot E, Jacobs S, Mouginot J and Scheuchl B (2013) Ice-shelf melting around Antarctica. Science, 341(6143), 266-270 (doi: 10.1126/Science.1235798)

Schytt V (1958) The inner structure of the ice shelf at Maudheim as shown by core drilling. In Norwegian-British-Swedish Antarctic Expedition, 1949-52, scientific results. Norsk Polarinstitutt, Oslo, 113-151

Somaraju $\mathrm{R}$ and others (2006) Frequency, temperature and salinity variation of the permittivity of seawater. IEEE Trans. Antennas Propag., 54(11), 3441-3448

United States Geological Survey (USGS) (2012) Landsat Image Mosaic of Antarctica (LIMA). lima.usgs.gov/ 